\title{
Exploring Twitter Interactions through Visualization Techniques: Users Impressions and New Possibilities
}

\author{
Guilherme Coletto Rotta, Vinícius Silva de Lemos, Ana Luiza Moura da Cunha, \\ Isabel Harb Manssour, Milene Selbach Silveira, and André Fagundes Pase \\ Faculdade de Informática, Pontifícia Universidade Católica do Rio Grande do Sul, \\ Av. Ipiranga 6681, Prédio 32, \\ 90619-900, Porto Alegre, RS, Brazil \\ \{guilherme.rotta, viniciusdelemos, analza.cunha\} @gmail.com, \\ \{isabel.manssour, milene.silveira, afpase\} apucrs.br
}

\begin{abstract}
Social networking websites have been taking a huge space on the Internet. With millions of members spread across the world, these services connect their users through "friendship" relations, and within a short period of time the information is disseminated in the network. Considering Twitter, we investigated how standard techniques of information visualization can improve the analysis of its users' behavior, i.e. the group of people who most closely relate, and the frequency and type of messages they send. This investigation was done through user's studies with a set of visualization techniques implemented in an interactive system prototype.
\end{abstract}

Keywords: visualization techniques, social networks, twitter users' behavior.

\section{Introduction}

The advent of Web 2.0 has aroused the emersion of new services for the Internet over recent years, giving users a higher level of - user-to-user - interaction in the network. Within this context, several applications focused on the relationship among users were developed, created to bring together people with specific interests in common, to facilitate the contact between distant friends and, for instance, the contact between a company and its customers. Social networks are, mostly, websites created with these purposes, and have millions of members spread around the world [1]. With the everincreasing number of members, a large amount of data is created on these networks and flows over the Internet. Making an analysis of this information can be difficult, since the complexity and the way it is organized do not assist in this task.

Taking into account the amount of information published in these networks, and the growing speed in which this information is updated and exchanged, the research herein presented focuses on how standard and interactive techniques of information visualization can help in the relation and extraction of new information based on what has already been published. 
Considering Twitter, it differs from other social networking sites because a friendship link doesn't need to be accepted by both parts: in order to receive tweets from a user, a person must follow him/her; however, the user is not obliged to follow this person back. Since Twitter just allows you to view the friends of one member at a time, and through a simple list that appears on every profile page, information visualization techniques can help to better view the network of contacts, and to analyze the frequency that individuals or groups send tweets and what kind of tweets they send the Twitter Users' Behavior.

In order to investigate this, we made user studies using a system prototype developed in our University, which provides visualization techniques that allow the analysis of users' connections, and the frequency of tweets sent by one or a group of users, and, mainly, it provides ways to classify these tweets, a feature difficult to find in most existing visualization tools for Twitter. Thus, as our main contribution, we demonstrate how interesting for the user point of view (given different user profiles) is to allow an integrated manner to explore and analyze the interactions of one user or a group of users in a social network.

This next section presents the visualization techniques available in the prototype. The user studies and a discussion on how they can help to analyze users' behavior are following presented. Some conclusions and future works are presented in the last section.

\section{Visualization Techniques to Analyze Users' Behavior}

There are many tools that use information visualization techniques to help in the analysis of users' behavior, as presented in some previous works [2-5]. In the work herein presented, we used the prototype system developed by Rotta et al. [6], called DeepTwitter.

DeepTwitter provides standard visualization techniques, selected according to some related work [7-9], to allow the understanding of how they can help in the users' behavior research in social networks. Through them, users' connections and the frequency of tweets sent by one or a group of users can be easily analyzed. Tag cloud visualization, most popular users and ways to classify the tweets are also provided, enabling the learning about the social network and the behavior of its members, through a constant collection of generated data.

Figure 1 shows DeepTwitter's main window, which displays the graph of the user's social network, expanded as he/she selects members of the network to include their friends or followers to the network. Two groups of users can be also observed in Figure 1, as well as a window that quickly shows some information regarding the user on which the mouse is over, such as his/her last tweet, description, number of friends and followers, etc. This visualization plays an important role, given that it has the ability to display, at once, a large number of members and connections, a task not possible to be done through the website. The discovery of other members that the user 
might have interest in also occurs in a very efficient way, through the verification of which users related to him are already following these members, for example.

The prototype also enables the creation of categories, into which different tags can be inserted. Through these tags tweets could be classified in both, timeline and tag cloud visualizations. Thus, it is possible to differentiate tweets by subject, clustering all the tweets that contain a given set of tags inside the same category that holds these tags.

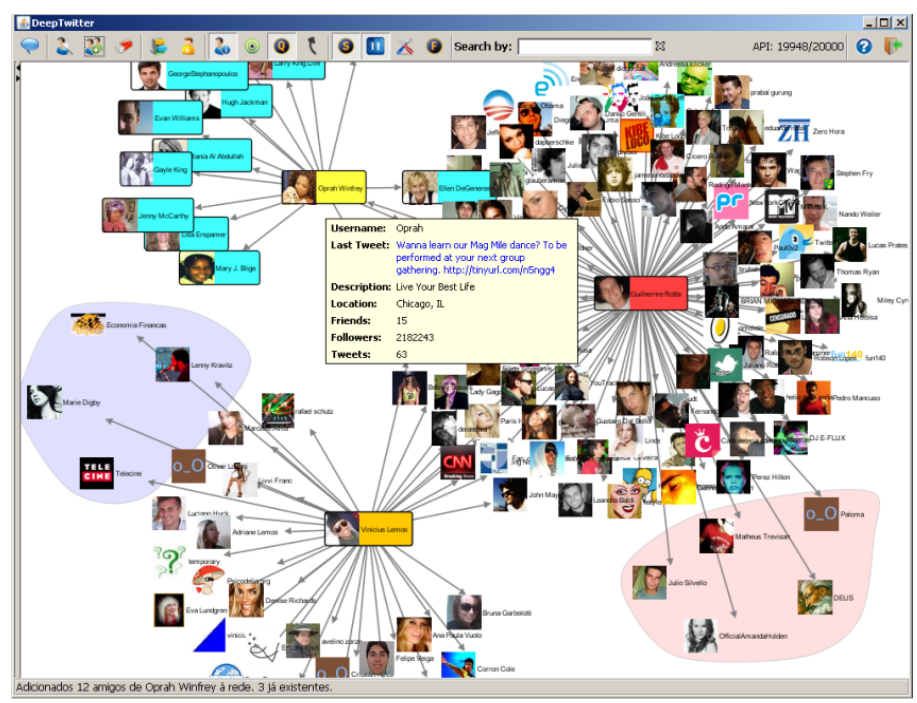

Fig. 1. The user social network with two groups of users and a window containing information about the selected user

When visualizing tweets from individuals or groups in DeepTwitter's main window it is possible to open the timeline visualization. The timeline organizes the tweets on a chart, based on the day (x axis) and time (y axis) in which they were sent, allowing the observation of a user or group of users behavior, such as the frequency in which the tweets are sent and what times and subjects are predominantly devoted to it. For timeline visualization, users can choose: the option to display the users' pictures or ellipses for each tweet; and the option to classify or not tweets within the categories already defined. By doing this, the pictures are outlined or the ellipses are filled with different colors, in order to facilitate the identification of which tweets belong to each category. Moreover, the tags are highlighted when the mouse moves across the tweet, as shown in Figure 2.

DeepTwitter also provides a tag cloud visualization, which shows the tags with different font sizes to allow the user to quickly and easily find information [10]. The tags could be the ones specified by the user when creating categories, or the ones that are currently the Trending Topics at the Twitter homepage. 


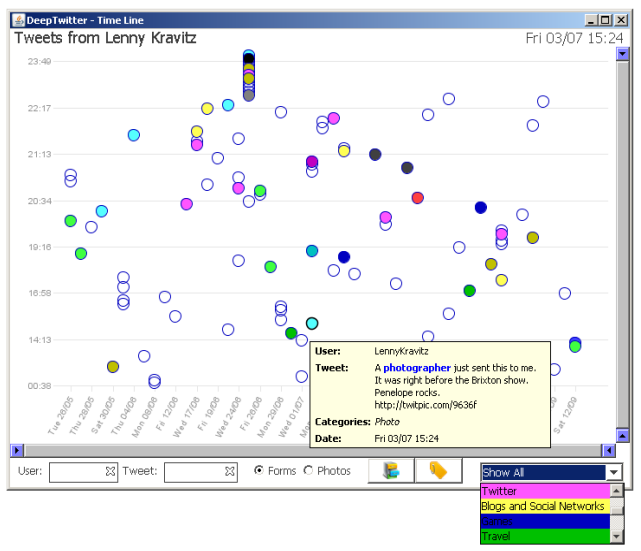

Fig. 2. Example of a Timeline with tweets classification

Finally, the most popular user visualization can be applied to a group of selected members or to all users in the contact network. The classification can be made by the number of friends, followers, favorite tweets, or tweets that have already been written. When one of these options is selected through a button interface, the picture size is appropriately changed in relation to the selected option and to other users. According to the selected option, the user that has more friends, e.g., appears with the biggest picture, and the one that has fewer friends, appears with the smallest picture. Through this visualization, with just a quick look the user can identify the most active or important members from his network of contacts, which would be very difficult to do using the Twitter website.

\section{Getting User Impressions about the Visualization Techniques}

In order to verify users' impressions about the use of visualization techniques, and to know which public would be more interested in this kind of tool, an user study was conducted, in two phases: the first one focusing on the general public, characterized by people who use Twitter for personal use (without professional goals), and the second focused on professionals who use Twitter as support for their work or even as their main activity (as professionals who monitor the impact of their companies on social networks and the Internet, for example).

In both studies, aiming to allow the users to understand the visualization techniques and their possibilities of use in a real environment, they were invited to use the prototype in their day-to-day activities. To better understand and analyze this use, a qualitative approach [11], with a restricted number of users was chosen: first, the users were invited to use the prototype and, then, to answer a set of questions related to this use. 


\subsection{First Phase: General Public}

The methodology used in the first phase and its results are presented as follows.

Used Methodology. In this phase, typical Twitter's users from the general public were invited to participate in the study. Following the example of the evaluations of the related work [2-4], which were performed respectively with 10,12 and 12 users, we invited 11 users to use DeepTwitter and then answer an online questionnaire about that use. The online questionnaire contained 21 open and closed questions about the user profile and his experience with Twitter and DeepTwitter.

By agreeing to participate, the users received a copy of the prototype and were free to use any visualization technique available from the tool for about a week and at any time they so wished. It is worthwhile mentioning that this use was made in an individual way and it was not accompanied by any observer. After this use they were instructed to answer the online questionnaire. The questionnaire contained open and closed questions, about the user profile, the Twitter and the prototype.

Results. Most users that answered the questionnaire were students (82\%) and from the Social Communication area (55\% of the students).

Regarding the use of Twitter, we found that most users (91\%) had used it for two years or more and $64 \%$ used it every day. When questioned about why they used this social network, the main choices were (the users could choose more than one option): personal purposes $(64 \%)$, work (36\%) and information (36\%).

Regarding the use of DeepTwitter, $64 \%$ used it for one week, mostly using the prototype less than once a day (64\%). In relation to the visualization tools available in it, the Social Networking Visualization was the most popular tool, chosen by $64 \%$ of the users when asked about the main advantage of using DeepTwitter and by $73 \%$ when asked about the visualization tool they considered the most interesting. The possibility to classify the tweets was also pointed out by $27 \%$ of users.

The preference for these two visualization tools - social networking and tweets classification - was also highlighted in the users comments: "This visualization facilitates the verification of users who I follow that also follow me, which is very interesting", "The social networking is cool because you can check nearby connection; the tweets per group also facilitate some 'conversations", and "tweets classification per tag is a good function for organizing content".

Beyond the scope of personal use, one possible application was highlighted by two distinct users: the use by companies. One of the users pointed out that for a company this Social Networking visualization tool would be very useful for the connection with potential new followers: "Especially for a business twitter, this tool helps you connect to new followers that have to do with the company subject. For example, through the social networking visualization I can see who are the followers in common among my followers, it helps me follow relevant people in my twitter". Another user proposes the use of tags classification for this purpose: "for a company, for example, launching a promotional tag". 


\subsection{Second Phase: Professionals}

The methodology used in the second phase and its results are presented as follows.

Used Methodology. In line with the results of the first phase that pointed to a professional use of this kind of tool, we developed a second phase with professionals who use Twitter as a working tool, inviting them to freely use the prototype in their day-today work, and after this use, participate in an interview to report their impressions.

As occurred in the previous phase, the participants received a copy of the prototype to use, by themselves and without the help of an observer, for about a week. The three groups of participants in this phase were professional users, as will be described in the next section. After using the prototype, they participated in a semi-structured interview, conducted by members of the research team. The interview began with 14 questions about the user profile and his experience with Twitter and DeepTwitter.

Results. The participants interviewed represent three distinct profiles: (1) employees of a private company of web hosting that were responsible for the company's public relations (group composed by 3 participants, that answered the questions in group and used the tool with the company account; they will be identified here as User 1); (2) the team responsible for the Twitter account of the Faculty of Social Communication of a large private university in the country (group composed by about 4-6 participants, students of the University, who also responded in group and used the institutional account, so they will be identified here as User 2); and (3) a University professor of Journalism (User 3), who used the program with his Twitter account. The academic profiles of users covered the areas of Public Relations, Journalism and Administration, and their professional profiles are about Social Communication and Customer Relations.

Focusing specifically on the last part of the interview, the users connected to the area of relationships (Users 1 and 2), when asked about the main feature of the prototype, highlighted the Visualization of their followers. User 1 highlights that with the timelines they can "identify over time followers of our activities in order to be aligned with our audience". User 3, working with Journalism, believes that the main feature is "the ability to follow the conversations of specific groups of people". These points were confirmed when all users of this phase highlighted that the visualization tool they would use more often would be the timeline analysis. User 3 said: "For journalism or marketing, for example, the analysis of the timeline is a very useful feature".

\subsection{Discussion}

Some of the issues presented in the results will be discussed in accordance with the visualization techniques available.

Social Network Visualization. From our studies, we found that for users of the general public the Social Network Visualization was considered the most attractive feature. On the other hand, users from companies (professionals), who often focus on 
their followers' behavior, highlighted that this feature should be removed from the prototype because the visualization gets very confusing when showing a great number of followers. That is, to the general public this feature allows a very interesting view of their entire network of contacts (and the relationships between their followers and who they follow), while for users interested mainly in their followers' activity, with hundreds (and even thousands) of them, this way of viewing the network seemed useless, because the amount of users shown did not allow a clear view of these connections. In this case, alternative techniques for the visual analysis of large graphs [12] could be presented, but that was out of the scope of this research.

Timeline and Tweets Classification. To users concerned with the relationship area, the timelines allow them to monitor the activities of their followers and thus find a better way to interact with them, knowing the best time of the day to send them an important notification or greeting message, for instance. From a journalistic point of view, the possibility of viewing the timelines from user groups or the classification of tweets permits focusing on specific issues (or conversations), enabling the analysis of new perspectives (how a particular post was discussed, how a particular group reacted to certain news, and so on), thus generating new information ("news").

Tag Cloud and Most Popular Users. Since Tag Cloud and Most Popular Users are visualizations techniques very common nowadays, these were the least mentioned in the evaluation. Only one user highlighted the possibility of using the tag cloud for mapping the subjects most in vogue at the moment. Another one mentioned that the most popular users could be used in order to evaluate the influence of their followers.

\section{Conclusion and Future Works}

The increase in use of social networks has been intense in recent years, as intense as the activity of users on these networks. Understanding the behavior of users on these networks, e.g. to companies' public relations identify a better way to get in touch with them, can be a hard task, given their high level of interactivity. In this context, we discussed how information visualization techniques could be helpful in supporting this task, through a set of standard visualization techniques and the discussion of these visualizations with its potential users.

From the analyses carried out we can clearly see that different profiles of users are interested in different features (and in different visualization ways), as noted with the preference of users in general (general public) for the social network visualization, and professional users for the timelines. In addition, a comment from one of the users (professional profile) that believes that everything the Twitter already offers should be excluded from the prototype led us to reflect.

Adding to this reflection the trend of users to simultaneously use several small applications to monitor the use of social networks, we now are designing distinct small visualization applications, focusing on specific market niches. Our first application is 
aimed specifically at professionals working in the area of relationships and their needs, exploring features as visualization of feelings and statistics about mentions.

Focusing on the information visualization area, the interest of users in the visual exploration of the available information opens the opportunity to research and develop novel interactive visualization techniques, focused specifically on social networks, and, also, in multi touch devices that are widely used nowadays and could provide new interactive approaches to visualization.

Acknowledgments. This work was partially supported by PUCRS (Edital 07/2012 Programa de Apoio a Integração Entre Áreas/PRAIAS).

\section{References}

1. Boyd, D.M., Ellison, N.B.: Social Network Sites: definition, history, and scholarship. J. Computer-Mediated Communication 13(1), 11 (2007)

2. Barata, G., Nicolau, H., Gonçalves, D.: AppInsight: What have I been doing? In: International Working Conference on Advanced Visual Interfaces, pp. 465-472 (2012)

3. Leung, C.K.-S., Carmichael, C.L.: Exploring Social Networks: a frequent pattern visualization approach. In: 2nd International Conference on Social Computing, pp. 419-424 (2010)

4. Marcus, A., Bernstein, M.S., Badar, O., Karger, D.R., Madden, S., Miller, R.C.: TwitInfo: aggregating and visualizing microblogs for event exploration. In: Conference on Human Factors in Computing Systems, pp. 227-236 (2011)

5. Kaye, J., Lillie, A., Jagdish, D., Walkup, J., Parada, R., Mori, K.: Nokia Internet Pulse: a long term deployment and iteration of a Twitter visualization. In: Conference on Human Factors in Computing Systems, pp. 829-844 (2012)

6. Rotta, G., Lemos, V., Lammel, F., Manssour, I., Silveira, M., Pase, A.: Visualization Techniques for the Analysis of Twitter Users' Behavior. In: 7th International AAAI Conference on Weblogs and Social Media (2013)

7. Heer, J., Boyd, D.: Vizster: Visualizing online social networks. In: IEEE Symposium on Information Visualization, pp. 32-39 (2005)

8. Perer, A., Shneiderman, B.: Integrating statistics and visualization: case studies of gaining clarity during exploratory data analysis. In: SIGCHI Conference on Human Factors in Computing Systems, pp. 265-274 (2008)

9. Kwak, H., Hong, Y., You, J., Moon, S.: Visualizing a Personal Timeline By Adding Multiple Social Contexts. In: International AAAI Conference on Weblogs and Social Media, pp. 14-17 (2012)

10. Halvey, M.J., Keane, M.T.: An assessment of tag presentation techniques. In: 16th International Conference on World Wide Web, pp. 1313-1314 (2007)

11. Denzin, N.K., Lincoln, Y.S.: Collecting and Interpreting Qualitative Materials. Sage Publications, Inc., London (2007)

12. von Landesberger, T., Kuijper, A., Schreck, T., Kohlhammer, J., Wijk, J.J., van, F.J.D., Fellner, D.W.: Visual Analysis of Large Graphs: State-of-the-Art and Future Research Challenges. Computer Graphics Forum 30(6), 1719-1749 (2011) 